\title{
Curva de crescimento de ovinos Santa Inês no Vale do Gurgueia
}

\author{
Growth curve Santa Inês sheep in the Valley Gurguéia
}

\author{
Ó, Alan Oliveira do ${ }^{1 *}$; RÊGO NETO, Aurino de Araújo ${ }^{1}$; SANTOS, Gleyson Vieira \\ dos $^{1}$; SARMENTO, José Lindenberg Rocha ${ }^{2}$; BIAGIOTTI, Daniel ${ }^{1}$; SOUSA, José \\ Ernandes Rufino de ${ }^{3}$
}

\author{
${ }^{1}$ Universidade Federal do Piauí, Teresina, Piauí, Brasil. \\ ${ }^{2}$ Universidade Federal do Piauí, Departamento de Zootecnia, Bom Jesus, Piauí, Brasil. \\ ${ }^{3}$ Universidade Federal Rural do Semi-Árido, Departamento de Ciência Animal, Mossoró, Rio Grande do \\ Norte, Brasil. \\ *Endereço para correspondência: alanoo1@hotmail.com
}

\section{RESUMO}

Objetivou-se descrever o crescimento de ovinos Santa Inês no Vale do Gurgueia a partir da análise de modelos não lineares: Brody,Von Bertalanffy, Richards, Logístico e Gompertz. Após a definição do modelo de melhor ajuste, avaliou-se a influência de fatores ambientais (estação de nascimento, sexo, tipo de parto e idade da mãe ao parto) sobre os parâmetros da curva. Foram utilizados dados do desenvolvimento ponderal de 61 ovinos, do nascimento aos 350 dias de idade, no período de janeiro de 2009 a dezembro de 2010. Os parâmetros foram estimados por meio do NLIN do SAS. Os modelos de Von Bertalanffy, Richards, Logístico e Gompertz ajustaram bem os dados de crescimento, entretanto, os modelos de Brody e Richards apresentaram maior variação no Quadrado Médio dos Resíduos. Com base no gráfico de dispersão dos resíduos, constatou-se que o modelo de Gompertz apresentou ajuste ligeiramente superior ao modelo de Von Bertalanffy. A taxa de crescimento absoluto estimada demonstrou crescente até os 28 dias de idade. $\mathrm{O}$ efeito de sexo foi importante fonte de variação para o parâmetro A, bem como os efeitos de idade da mãe ao parto aos parâmetros B e K. As correlações estimadas entre os parâmetros A e K, $\mathrm{B}$ e $\mathrm{K}$ foram negativas. Efeitos ambientais mostraram-se importantes fatores que influenciam os parâmetros da curva de crescimento de ovinos Santa Inês no estado do Gurgueia.

Palavras-chave: efeito de ambiente, modelo não linear, ovinos deslanados, peso à maturidade, taxa de crescimento.

\section{SUMMARY}

The objective of this study was to analyze nonlinear models between Brody, Von Bertalanffy, Richards, Logistic and Gompertz, that best fit to describe the growth of Santa Ines sheep in Valley Gurguéia and, after setting the best model, it was to evaluated the influence of environmental factors (season of birth, sex, type of birth and mother's age at birth) on the parameters of the curve. Data were obtained from 61 sheep weight development from birth to 350 days of age from January 2009 to December 2010. The parameters were estimated using the SAS NLIN procedure. The models of Von Bertalanffy, Richards, Logistic and Gompertz fit the data well for growth, however, greater variation in Mean Square Waste was seen for Brody and Richards models. Based on the scatter plot of the waste, it was found that the Gompertz model presented set slightly higher than the model of von Bertalanffy. The estimated rate of absolute growth showed increased up to 28 days old. The effect of sex was an important source of variation for the parameter A, as well as the effects of age of mother at birth to the parameters B and K. The estimated correlations between the parameters A and $\mathrm{K}, \mathrm{B}$ and $\mathrm{K}$ were negative. Environmental effects were important factors influencing the parameters of the growth curve of Santa Inês sheep in the state of Gurguéia.

Keywords: environmental effect, growth rate, hair sheep, non-linear model, weight maturity. 


\section{INTRODUÇÃOO}

As análises de dados de medidas repetidas são indispensáveis na produção animal, pois incluem as situações em que as unidades experimentais ou indivíduos, de diferentes subpopulações ou tratamentos (sexo, raça, entre outros), são analisados ao longo de diversas condições de avaliação (peso, ano, idade) - (FREITAS, 2005). Funções não lineares, com parâmetros biologicamente interpretáveis e desenvolvidas empiricamente para relacionar peso e idade, são as mais utilizadas para modelar a curva de crescimento dos animais (LÔBO et al. 2006). Nesse sentido, as funções não lineares podem ser utilizadas para descrever o crescimento do animal ao longo do tempo e possibilitar a avaliação dos fatores genéticos e de ambiente que influenciam a forma da curva de crescimento (PACHECO, 2008).

No Brasil, são vários os estudos que tratam da aplicação de modelos não lineares para o ajuste de curvas de crescimento em ovinos (McMANUS et al. 2003; GUEDES et al., 2004; FREITAS, 2005; SARMENTO et al. 2006b ; LÔBO et al. 2006; MALHADO et al. 2008; MALHADO et al. 2009; SOUZA et al., 2011). Quando se buscam trabalhos realizados no Piauí, se encontra uma literatura escassa no que diz respeito ao estudo da curva de crescimento em rebanhos Santa Inês da região. Dessa forma, pesquisas que avaliem o desempenho de animais adaptados às condições locais para características de importância econômica são imprescindíveis para a utilização desse recurso genético em benefício da sociedade (SOUZA et al., 2011).
O efeito de ambiente pode ser confundido com o genético, quando os animais que foram submetidos à diferentes condições de clima e manejo são comparados diretamente. Para incrementar a acurácia do processo seletivo, os critérios de seleção devem ser adequadamente ajustados para os efeitos de ambiente (DAL-FARRA et al., 2002). O entendimento de tais fatores é imprescindível para o crescimento dessa atividade pecuária, haja vista as características relacionadas ao crescimento do animal geralmente sofrerem influências de fatores genéticos e ambientais (FIGUEIREDO FILHO et al., 2012).

Assim, objetivou-se comparar diferentes modelos não lineares para descrever a curva de crescimento e avaliar a influência de alguns fatores de ambiente sobre os parâmetros do modelo que melhor se ajustou à curva em ovinos Santa Inês.

\section{MATERIAL E MÉTODOS}

Os dados aqui utilizados foram originados do controle de desenvolvimento ponderal de 57 cordeiros (24 machos e 33 fêmeas). Os animais nasceram no Núcleo de Seleção e Conservação de Recursos Genéticos de Ovinos Deslanados para Produção de Carne no Sul do Piauí, pertencente à Universidade Federal do Piauí (UFPI), Campus Professora Cinobelina Elvas (CPCE), em Bom Jesus-PI, entre os meses de janeiro de 2009 e dezembro de 2010.

Os ovinos foram submetidos ao sistema semi-intensivo de manejo. As crias foram mantidas junto às mães até os 60 dias de idade. Durante todo ano, os animais recebiam, no cocho, suplementação alimentar à base de 
capim-elefante (Penisetum purpureum) picado, feno de leucena (Leucaena leucocephala Lam), silagem de milho (Zea mays) e ração concentrada. A pastagem cultivada era formada por andropogon (Andropogon gayanus).

No manejo reprodutivo, as coberturas foram efetuadas por meio de monta natural controlada. A coleta de dados compreendeu informações de identificação da ovelha e do carneiro, data da cobertura; data do nascimento; sexo; tipo de nascimento; peso ao nascer; idade da mãe ao parto e pesos, normalmente tomados ao nascimento aos 350 dias de idade, em intervalos de aproximadamente 14 dias. Foram utilizados cinco modelos não lineares, descritos na Tabela 1 , para verificar o que melhor descreveu a curva de crescimento média do rebanho.

Tabela 1. Forma geral dos modelos não lineares

\begin{tabular}{lcl}
\hline Modelo & \multicolumn{2}{c}{ Forma Geral } \\
\hline Gompertz & $Y=A e^{-B e^{-k t}}+\varepsilon$ & (LAIRD, 1965) \\
Brody & $Y=A\left(1-B e^{-K t}\right)+\varepsilon$ & (BRODY, 1945) \\
Von Bertalanffy & $Y=A\left(1-B e^{-K t}\right)^{3}+\varepsilon$ & (BERTALANFYY, 1947) \\
Logístico & $Y=A\left(1+B e^{-K t}\right)^{-1}+\varepsilon$ & (NELDER, 1961) \\
Richards & $Y=A\left(1-B e^{-K t}\right)^{M}+\varepsilon$ & (RICHARDS, 1959) \\
\hline
\end{tabular}

Como se observa na Tabela $1, Y$ é o peso corporal à idade t; $A$, o peso assintótico quando $\mathrm{t}$ tende a mais infinito, ou seja, esse parâmetro é interpretado como peso à idade adulta; $B$, uma constante de integração, relacionada aos pesos iniciais do animal e sem interpretação biológica bem definida. $\mathrm{O}$ valor de $B$ é estabelecido pelos valores iniciais de $Y$ e t; $k$ é interpretado como taxa de maturação, entendida como indicador da velocidade com que o animal se aproxima do seu tamanho adulto; e $M$ é o parâmetro que dá forma à curva. Sua fixação determina a forma desta e, consequentemente, o ponto de inflexão.

Assim, ao assumir o ponto de inflexão do modelo de Richards igual a 0 , obtém-se o modelo de Brody; quando igual a 2/3, o modelo Von Bertalanffy; próximo a 1, o modelo de Gompertz; e se igual a 2, o modelo Logístico. Com isso, percebe-se que os primeiros são casos especiais do modelo Richards, que possui quatro parâmetros.

Os parâmetros dos modelos foram estimados pelo método iterativo de Gauss Newton por meio do NLIN do SAS (2000). O critério de convergência adotado foi $10^{-8}$ e os critérios utilizados para descrever o melhor modelo foram: Quadrado Médio do Resíduo (QMR); Coeficiente de determinação $\left(\mathrm{R}^{2}\right)$; Desvio médio absoluto dos resíduos (DMA) e análise gráfica dos resíduos. O Quadrado Médio do Resíduo (QMR) foi calculado ao se dividir a soma de quadrados do resíduo, obtida pelo SAS, pelo número de observações, que é o estimador de máxima verossimilhança da variância residual, para que se pudesse comparar o QMR dos diferentes modelos por possuírem número diferentes de parâmetros a serem estimados. O Coeficiente de determinação $\left(\mathrm{R}^{2}\right)$ foi calculado como o quadrado da correlação entre os pesos observados e estimados, que é equivalente a 
$1-\left(\frac{S Q R}{S Q T c}\right)$, em que $S Q R$ é soma de quadrados do resíduo e SQTc, a soma de quadrados total corrigida pela média (SARMENTO et al., 2006b). O Desvio médio absoluto dos resíduos (DMA) foi a estatística aqui proposta para avaliar a qualidade de ajuste, calculado como a seguir: $D M A=\sum_{i-1}^{n}\left(\frac{Y_{i}-\hat{Y}_{i}}{n}\right)$ em que $Y_{i}$ é o valor observado, $\hat{Y}_{i}$ o valor estimado, e $n$ o tamanho da amostra (SARMENTO et al., 2006b). Quanto menor o valor do DMA, melhor o ajuste. Por fim, a análise gráfica dos resíduos, proposta por Sarmento et al. (2006b), foi utilizada para verificar, entre os modelos escolhidos, quais subestimam ou superestimam os pesos médios observados e estimados.

Escolhido o melhor modelo, calculou-se a taxa de crescimento absoluta (TCA), obtida a partir da primeira derivada do modelo ajustado em relação ao tempo $(\partial \mathrm{Y} / \partial \mathrm{t})$. A TCA é, na realidade, o ganho de peso obtido por unidade de tempo. Como o tempo está registrado em dias, representa o ganho de peso médio diário, estimado ao longo da trajetória de crescimento, ou seja, a taxa média de crescimento dos animais dentro da população (SARMENTO et al., 2006b).

Foram ainda estimados os pesos do nascimento aos 350 dias de idade por meio da função escolhida. Os parâmetros da curva estimados para cada animal, a depender do modelo ajustado, foram analisados pelos procedimentos GLM e CORR do SAS (2000) para determinação da influência de efeitos de ambiente e das correlações entre os parâmetros estimados.

Os efeitos de ambiente utilizados na análise foram: estação de nascimento, agrupada em chuvosa (outubro a abril) e seca (maio a outubro); idade da mãe ao parto, agrupadas em classes de idades (classe $1=$ fêmeas adultas com idades até dois anos; classe 2 = fêmeas com idades superiores a dois e menores ou iguais a três anos; classe $3=$ fêmeas com idades superiores a três anos e menores ou iguais a quatro anos de idade e classe $4=$ fêmeas com idades superiores a quatro anos); tipo de nascimento (simples e duplo) e sexo do animal.

O modelo estatístico adotado para verificar os fatores de ambiente que poderiam influenciar a forma da curva de crescimento foi: $Y_{i j k l m}=\mu+T n_{i}+S_{j}+E_{k}+I M P_{l}+\varepsilon_{i j k l m} ;$ em que $Y_{i j k l m}$ é o vetor de observações (peso); $\mu$ é uma constante inerente às observações; $T n_{i}$ é o efeito do fixo do tipo de parto $i ; S_{j}$ efeito fixo do sexo $j ; E_{k}$ é o efeito fixo de estação de nascimento $k ; I M P_{l}$ é o efeito fixo de idade da mãe ao parto $l ; \varepsilon_{i j k l m}$ é o erro aleatório associado a cada observação. As médias estimadas por quadrados mínimos foram comparadas pelo teste de Tukey a 5\% de probabilidade.

\section{RESULTADOS E DISCUSSÃO}

A média de peso das crias ao nascimento (Tabela 2) foram próximas às encontradas por Malhado et al. (2008). Sarmento et al. (2006a) também registraram valores para peso ao nascer próximos aos aqui descritos $(3,61)$. Todavia, quando comparados aos demais pesos, os valores foram superiores aos apontados neste estudo (8,31 para P28 a e 19,49 para P112).

Com base no coeficiente de determinação (Tabela 2), observou-se que os maiores valores encontrados se apresentaram nos modelos de Von 
Bertalanfyy e Gompertz (0,982). Malhado et al. (2008) constataram que os melhores modelos utilizados para descrever a curva de crescimento, com base no $\mathrm{R}^{2}$, foram os modelos de Brody e Richards.

As diferenças entre os modelos de Gompertz e Von Bertalanffy foram mínimas para os valores de QMR e DMA (Tabela 3), o que permite afirmar que ambos se ajustaram de forma satisfatória aos dados de crescimento de ovinos Santa Inês. Ao observar os resultados presume-se que a avaliação da qualidade de ajuste em modelos não lineares, com base apenas no $\mathrm{R}^{2}$, pode não ser uma boa opção dado à semelhança de ajuste refletida por esta estatística (Tabela 3).

Sarmento et al. (2006b), quando analisaram o valor do DMA, constataram variações nos modelos de Brody e Logístico e que essas variações são imperceptíveis se observado apenas o $\mathrm{R}^{2}$. O mesmo fato foi constatado neste estudo.

Tabela 2. Média e desvios padrão dos pesos, observados ao nascimento (PN), peso aos 28 dias de idade (P28), aos 56 e seis dias (P56), pesos aos 112 dias (P112) e pesos aos 350 dias de idade (P350) de ovinos Santa Inês no Vale do Gurgueia

\begin{tabular}{llllll}
\hline Pesos & PN & P28 & P56 & P112 & P350 \\
\hline Média (kg) & 3,38 & 6,30 & 9,40 & 13,50 & 25,79 \\
Desvios Padrão (kg) & 0,86 & 1,93 & 2,88 & 4,07 & 7,08 \\
\hline
\end{tabular}

Tabela 3. Estimativa de Quadrado Médio do Resíduo (QMR), desvio médio absoluto (DMA) e coeficiente de determinação $\left(\mathrm{R}^{2}\right)$ para os modelos estudados

\begin{tabular}{lccc}
\hline \multirow{2}{*}{ Modelo } & \multicolumn{3}{c}{ Critérios } \\
\cline { 2 - 4 } & QMR & DMA & $\mathrm{R}^{\mathbf{2}}$ \\
\hline Brody & 1,786 & 0,963 & 0,973 \\
Bertalanffy & 1,169 & 0,818 & 0,982 \\
Gompertz & 1,175 & 0,817 & 0,982 \\
Logístico & 1,270 & 0,848 & 0,980 \\
Richards & 1,557 & 0,884 & 0,976 \\
\hline
\end{tabular}

O percentual de convergência dos modelos de Gompertz, Logístico e Von Bertalanffy foram semelhantes $(95 \%) \mathrm{e}$ superiores ao modelo de Brody $(78 \%)$ e Richards (31\%). Assim, constatou-se que quanto maior o número de convergências individuais melhor $\mathrm{O}$ modelo ajustado.

Os valores encontrados por Souza et al.(2011) foram superiores a $90 \%$ para todos o modelos estudados, portanto, diferente dos resultados encontrados neste trabalho. Sarmento et al. (2006b) afirmaram que modelo Richards não foi escolhido para representar a curva média, visto a diferença em qualidade de ajuste não superar a dificuldade em atingir convergência, possivelmente, por esse modelo necessitar de estimar um parâmetro a mais.

Observa-se que entre os modelos que se ajustaram de forma semelhante à curva média de crescimento (Figuras 1e 2), os modelos de Gompertz e Von 
Bertalanffy foram os que melhor se aproximaram da curva média de crescimento observada. Tal comportamento já era esperado, visto que, ao observar os demais critérios, os dois modelos foram os que melhor estimaram DMA e QMR (Tabela 2). No sentido oposto, Malhado et al. (2008) afirmaram que os modelos de Richards e Brody estimaram curvas semelhantes e que ambos superestimaram o crescimento dos animais em todas as idades, principalmente, dos 120 aos 330 dias de idade.

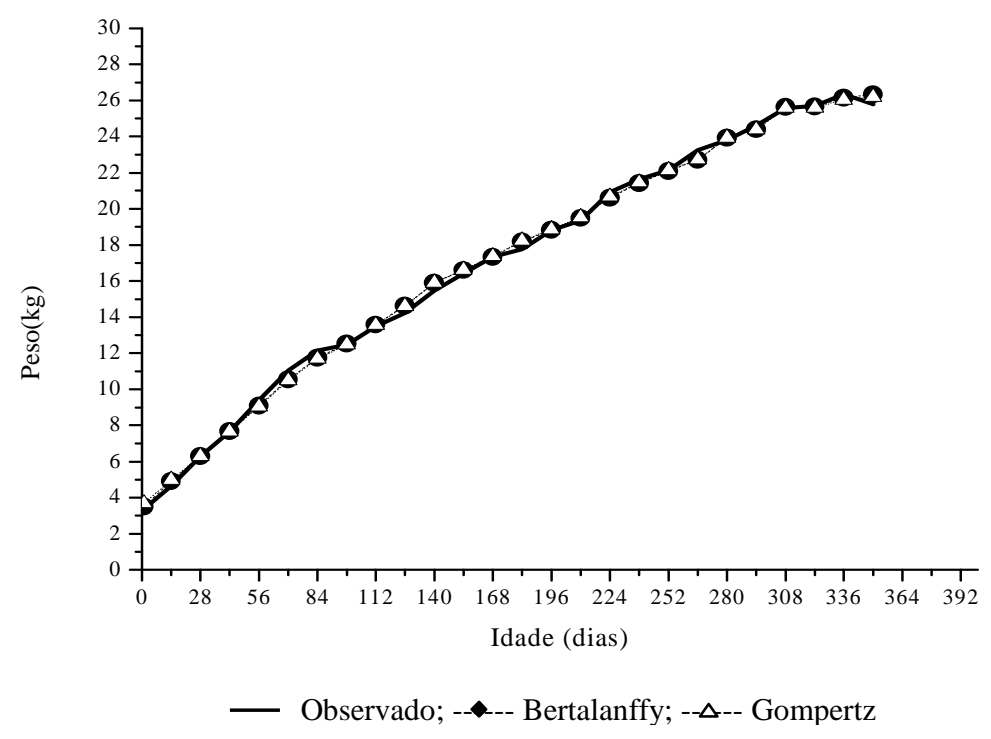

Figura 1. Peso em função das idades de acordo com os modelos de Von Bertalanffy, Gompertz, observado em ovinos da raça Santa Inês

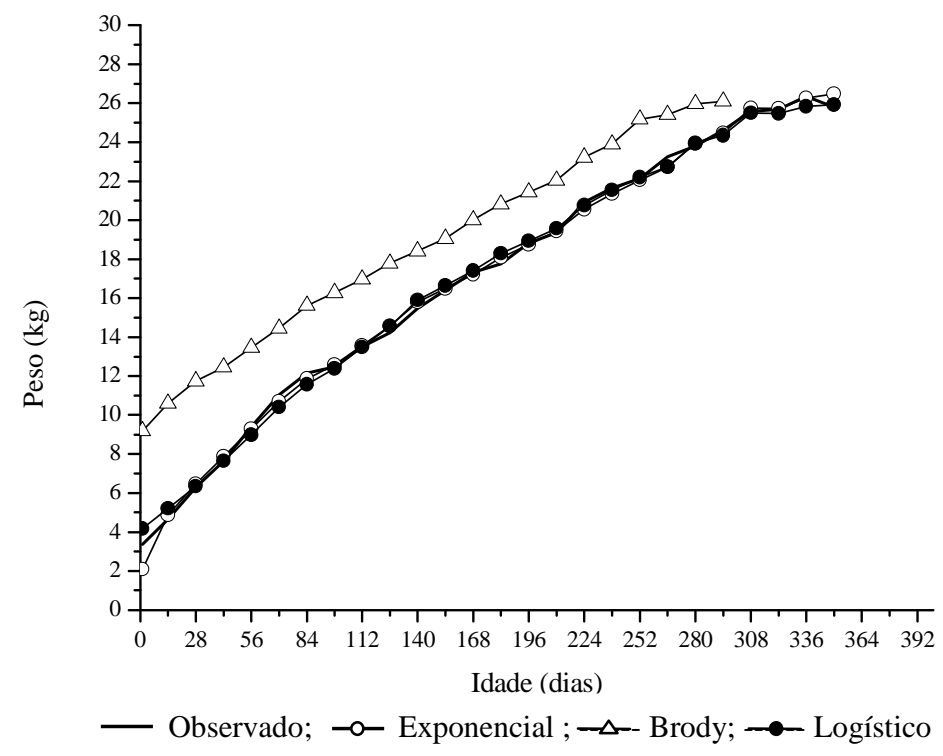

Figura 2. Peso em função das idades de acordo com os modelos Exponencial, Brody, Logístico, observado em ovinos da raça Santa Inês. 
Os maiores valores de resíduos foram encontrados na superestimação do peso ao nascer e na subestimação do peso aos 350 dias para o modelo de Von Bertalanffy (Figura 3). Dessa forma, presume-se que o modelo que melhor se ajustou para descrever a curva média de crescimento de ovinos Santa Inês foi o modelo de Gompertz.

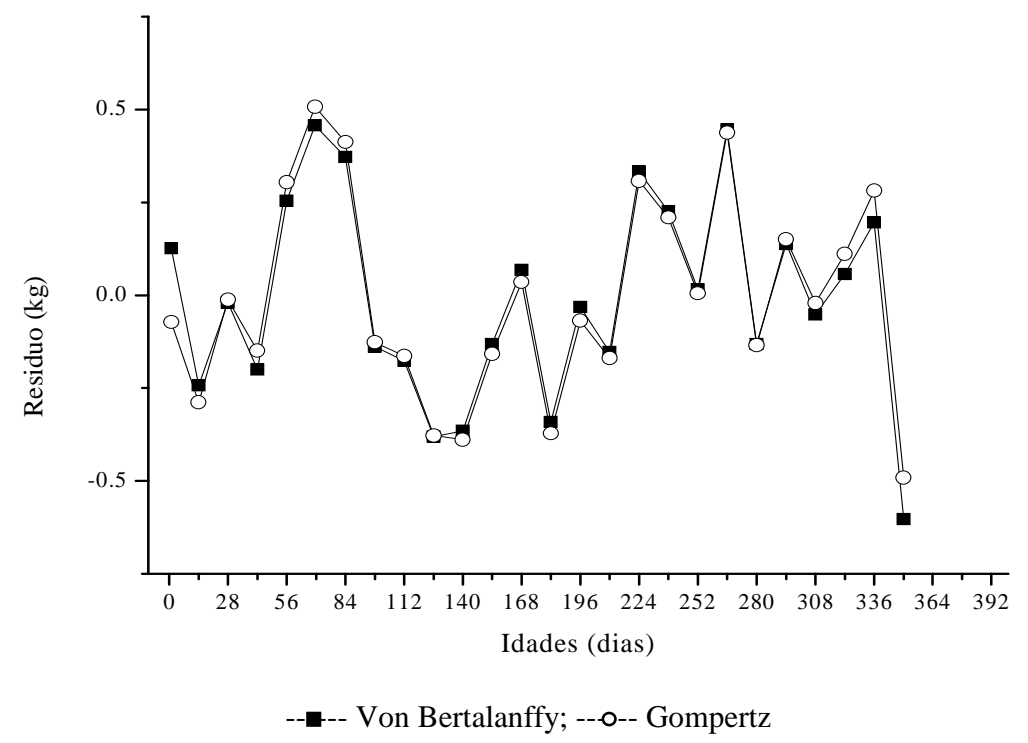

Figura 3. Dispersão dos resíduos estimados pelos modelos Gompertz e Von Bertalanffy de ovinos da raça Santa Inês

$\mathrm{Na}$ literatura, observou-se grande variação na escolha dos modelos que melhor ajustaram a curva de crescimento em ovinos. McManus et al. (2003) afirmaram que o melhor modelo para descrever a curva de crescimento no estudo foi o Logístico. Já Freitas (2005) complementa ao relatar que os modelos Logístico, Von Bertalanffy e Brody foram os mais versáteis para ajustar dados de crescimento de ovinos. Sarmento et al. (2006b) encontraram melhores ajustes pelo modelo de Gompertz. Enquanto Malhado et al. (2008) afirmaram que a função Logística é o modelo mais próximo do peso médio observado e, portanto, foi considerado o mais adequado para descrever o crescimento dos animais estudados. Essa divergência quanto aos diferentes modelos ajustados é teoricamente compreensível, pois irá depender do padrão de crescimento dos animais em estudo (SARMENTO et al. 2006b).

As taxas de crescimento absoluto (TCA), obtidas pela primeira derivada do modelo Gompertz em relação ao tempo, mostram que ela foi crescente, até atingir o máximo, em torno de $0,28 \mathrm{~kg} / \mathrm{dia}\left(K A e^{-1}\right)$, e depois decresceu com a idade (Figura 4). O ponto em que a função muda de crescente para decrescente, ou seja, o ponto de inflexão da curva foi atingido aos 28 dias de idade $\left(K^{-1}\right)$, com peso de aproximadamente $6,40 \quad \mathrm{~kg} \quad\left(A e^{-1}\right)$ (Figura 1). Os resultados obtidos indicam que a taxa máxima de crescimento foi alcançada muito cedo, antes dos dois meses de idade, e que a essa idade o animal já atingiu a velocidade máxima de crescimento. $\mathrm{O}$ ponto de inflexão é importante para 
auxiliar os produtores em programas alimentares específicos e na definição da melhor idade ao abate (SOUZA et al., 2011).

A TCA máxima para essa idade pode indicar limitação no crescimento, provavelmente, associada à disponibilidade insuficiente de alimentos. Após 56 dias de idade (desmame), observou-se que a taxa de crescimento continuou em declínio, possível reflexo de manejo inadequado que provoca uma menor capacidade de disponibilidade de nutrientes para atender à demanda, à medida que o animal cresce. Carneiro et al. (2009) afirmaram que as maiores TCAs foram verificadas no período pré-desmame, época em que a cria é dependente, principalmente, da produção de leite da matriz. Sarmento et al. (2006b) afirmam que o decréscimo da taxa de crescimento pode ser um indicativo de adaptação dos animais ao clima semiárido que os leva a adquirir esse padrão de crescimento.

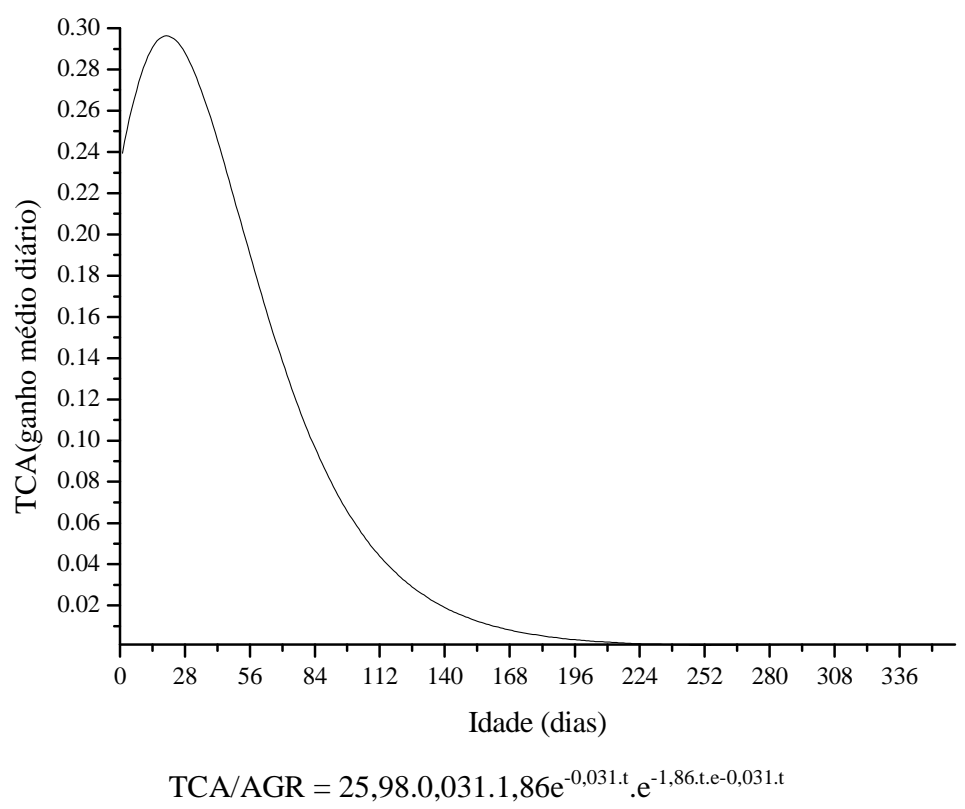

Figura 4. Comportamento da taxa de crescimento absolta estimada pelo modelo de Gompertz

Houve diferença significativa $(\mathrm{P}<0,05)$ para o efeito de sexo sobre o parâmetro A (Tabela 3). Sarmento et al. (2006b) observaram que o efeito sexo do animal influenciou de maneira expressiva os parâmetros A e $k$. A superioridade dos machos em relação às fêmeas talvez esteja vinculada ao dimorfismo sexual, o que representa maior peso dos machos à idade adulta. Barros et al. (2004); Furusho - Garcia et al. (2004) indicaram que os machos foram consideravelmente mais pesados que as fêmeas, quando mantidos em condições semelhantes de manejo e alimentação, durante a fase de desenvolvimento até a idade adulta.

A idade da mãe ao parto influenciou os parâmetros B e K da curva $(\mathrm{P}<0,05)$ (Tabela 4). Sarmento et al. (2006) encontraram efeito significativo da idade da matriz ao parto para os parâmetros A e K. Mudanças na produção de leite das matrizes e nos 
cuidados maternos por elas proporcionados podem influenciar significativamente o peso corporal dos cordeiros, bem como a velocidade com que as os mesmos atingem a idade adulta.

A correlação entre os parâmetros A e K foi significativa e negativa $(-0,26)$, o que corrobora os achados de McManus et al.(2003); Sarmento et al. (2006); Malhado et al. (2008). Uma provável explicação biológica para essa correlação seria que animais com elevadas taxas de crescimento têm menor probabilidade de atingir maiores pesos à maturidade do que aqueles que crescem lentamente no início da vida.

Tabela 4. Médias estimadas para os parâmetros A, B, K em função dos efeitos de estação de nascimento, sexo, tipo de nascimento e classe de idade da mãe ao parto pelo modelo de Gompertz

\begin{tabular}{lcccc}
\hline Efeitos & & $\mathrm{A}$ & $\mathrm{B}$ & $\mathrm{K}$ \\
\hline \multirow{2}{*}{ Estação } & Seca & $25,34^{\mathrm{a}}$ & $1,88^{\mathrm{a}}$ & $0,04^{\mathrm{a}}$ \\
& Chuvosa & $21,73^{\mathrm{a}}$ & $1,61^{\mathrm{a}}$ & $0,09^{\mathrm{a}}$ \\
\hline \multirow{2}{*}{ Sexo } & Macho & $28,86^{\mathrm{a}}$ & $1,79^{\mathrm{a}}$ & $0,07^{\mathrm{a}}$ \\
& Fêmea & $18,21^{\mathrm{b}}$ & $1,70^{\mathrm{a}}$ & $0,06^{\mathrm{a}}$ \\
\hline \multirow{2}{*}{ Tipo de nascimento } & Simples & $27,53^{\mathrm{a}}$ & $1,82^{\mathrm{a}}$ & $0,04^{\mathrm{a}}$ \\
& Duplo & $19,53^{\mathrm{a}}$ & $1,67^{\mathrm{a}}$ & $0,09^{\mathrm{a}}$ \\
\hline \multirow{3}{*}{ Idade da mãe ao parto } & 1 & $20,95^{\mathrm{a}}$ & $1,68^{\mathrm{ab}}$ & $0,07^{\mathrm{ab}}$ \\
& 2 & $24,64^{\mathrm{a}}$ & $1,68^{\mathrm{ab}}$ & $0,04^{\mathrm{ab}}$ \\
& 3 & $27,43^{\mathrm{a}}$ & $2,13^{\mathrm{a}}$ & $0,02^{\mathrm{b}}$ \\
& 4 & $21,13^{\mathrm{a}}$ & $1,50^{\mathrm{b}}$ & $0,12^{\mathrm{a}}$ \\
\hline
\end{tabular}

*Médias seguidas de mesma letra na coluna não diferem estatisticamente pelo teste Tukey $(\mathrm{P}>0,05)$.

O modelo Gompertz apresentou melhor distribuição residual e melhor ajuste na descrição da curva de crescimento, por isso foi escolhido entre os demais. Os efeitos de estação, sexo, e idade da matriz mostram-se importantes fatores não genéticos que influenciam os parâmetros da curva de crescimento de ovinos Santa Inês no estado do Gurgueia.

\section{AGRADECIMENTOS}

Conselho Nacional de Desenvolvimento Científico e Tecnológico (CNPQ) e ao Banco do Nordeste do Brasil.

\section{REFERÊNCIAS}

BARROS, N.N.; VASCONCELOS, V.R.; LOBO, R.N.B. Características de Crescimento de Cordeiros F-1 para abate no Semi-Árido do Nordeste do Brasil. Pesquisa Agropecuária

Brasileira, v.39, n.8, p.809-814 , 2004.

BERTALANFFY, L.V. Quantitative laws in metabolism and growth. The Quaterly Reviem of Biology, v.32, n.3, p.217-230, 1957.

BRODY, S. Bioenergetics and growth. New York: Reinhhold Publication, 1945. 
CARNEIRO, P.L.S.; MALHADO,

C.H.M; AFFONSO, P.R.A.M.; PEREIRA, D.G.; ZUZART, J.C.C.; RIBEIRO JUNIOR, M.; SARMENTO, J.L.R. Curva de crescimento em caprinos da raça Mambrina, criados na caatinga. Revista Brasileira de Saúde e Produção Animal [Online], v.10, n.3, p 536-545, 2009.

DAL-FARRA, R.A.; ROSO, V.M.; SCHENKEL, F.S. Efeitos de Ambiente e de Heterose sobre o ganho de peso do nascimento ao desmame sobre os escores visuais ao desmame de bovinos de corte. Revista Brasileira de

Zootecnia, v.31, n.3, p.1350-1361, 2002.

FIGUEIREDO FILHO, L.A.S.; SARMENTO, J.L.R.; CAMPELO, J.E.G.; SANTOS, N.P.S.; SOUSA, J.E.R.; BIAGIOTTI, D. Fatores ambientais e genéticos sobre a curva de crescimento de caprinos mestiços.

Comunicata Scientiae, v.3, n.3, p.154161, 2012.

FURUSHO-GARCIA, I.F.; PEREZ, J.R.O.; BONAGURIO, S.; ASSIS, R.M.; PEDREIRA, B.C.; SOUZA, X.R. Desempenho de cordeiros Santa Inês Puros e cruzas Santa Inês com Texel, Ile de France e Bergamácia. Revista Brasileira de Zootecnia, v.33, n.6, p.1591-1603, 2004.

GUEDES, M.H.P.; MUNIZ, J.A.; PEREZ, J.R.O.; SILVA, F.F.; AQUINO, L.H.; SANTOS, L.C. Estudo das curvas de crescimento de cordeiros das raças Santa Inês e Bergamácia considerando heterogeneidade de variâncias. Ciências Agrotécnicas, v.28, n.2, p.381-388, 2004.

FREITAS, A.R. Curvas de crescimento na produção animal. Revista Brasileira de Zootecnia, v.34, p.786-795, 2005.
LAIRD, A.K. Dynamics of relative growth. Growth, v.29, p.249-263, 1965.

LOBO, R.N.B.; VILLELA, L.C.V.; LOBO, A.M.B.O.; PASSOS, J.R.S.; OLIVEIRA, A.A. Parâmetros genéticos de características estimadas da curva de crescimento de ovinos Santa Inês.

Revista Brasileira de Zootecnia, v.35, p.1012-1018, 2006.

MALHADO, C.H.M; CARNEIRO, P.L.S; SANTOS, P.F; AZEVEDO, D.M.M.R.; SOUZA, J.C.; AFONSO, P.R.M. Curva de crescimento em ovinos mestiços Santa Inês x Texel criados no Sudoeste do Estado da Bahia. Revsista Brasileira de Saúde e Produção Animal [Online], v.9, n.2, p.210-218, 2008 .

MALHADO, C.H.M.; CARNEIRO, P.L.S.; MELLO, P.R.A.M.; SOUZA JUNIOR, A.A.O.; SARMENTO, J.L.R. Growth curves in Dorper sheep crossed with the local Brazilian breeds, Morada Nova, Rabo Largo, and Santa Inês. Small Ruminant Research, v.84, p.16-21, 2009.

McMANUS, C.; EVANGELISTA, C.; FERNANDES, L.A.C.; MIRANDA, R.M.; MORENO-BERNAL, F.E.; SANTOS, N.R. Curvas de crescimento de ovinos Bergamácia criados no Distrito Federal. Revista Brasileira de

Zootecnia, v.32, n.5, p.1207- 1212, 2003.

NELDER, J.A. The fitting of a generalization of the logistic curve. Biometrics, v.17, n.1, p.89-110, 1961.

PACHECO, A.; QUIRINO, C.R. Estudo das características de crescimento em ovinos. Pubvet, v.2, n.29, 2008.

Disponível em: http://www.pubvet.com.br/artigos_det.asp ?artigo=293. Acesso em: 11 jan. 2012. 
SARMENTO, J.L.R.; TORRES, R.A.; PEREIRA, C.S.; SOUSA, W.H.;

LOPES, P.S.; ARAÚJO, C.V.;

EUCLYDES, R.F. Avaliação genética

de características de crescimento de ovinos Santa Inês utilizando modelos de regressão aleatória. Arquivo Brasileiro de Medicina Veterinária e Zootecnia, v.58, n.1, p.68-77, 2006a.

SARMENTO, J.L.R.; REZAZZI, A.J.; SOUZA, W.H.; TORRES, R.A.; BREDA, F.C.; MENEZES, G.R.O. Estudo da curva de crescimento de ovinos Santa Inês.Revista Brasileira de Zootecnia, v.35, n.2, p.435-442, 2006 b.

SAS INSTITUTE. User's guide :

statistics. Version 8.1. Cary: NC, 2000.
RICHARDS, F.J. A flexible growth function for empirical use. Journal of Experimental Botany, v.10, p.290-300, 1959

SOUZA, L.A; CARNEIRO, P.L.S.; MALHADO, C.H.M.; PAIVA, S.R.; CAIRES, D.N.; BARRETO, D.L.F. Curvas de crescimento em ovinos da raça morada nova criados no estado da Bahia. Revista Brasileira de Zootecnia, v.40, n.8, p.1700-1705, 2011.

Data de recebimento: 06/03/2012

Data de aprovação: 20/09/2012 